\title{
Estimating the number of HIV infections averted: an approach and its issues
}

\author{
L M Heaton, ${ }^{1}$ R Komatsu, ${ }^{2}$ D Low-Beer, ${ }^{2,3}$ T B Fowler, ${ }^{1}$ P 0 Way ${ }^{1}$
}

${ }^{1}$ US Census Bureau, Washington, USA; ${ }^{2}$ The Global Fund To Fight AIDS, Tuberculosis and Malaria, Geneva, Switzerland; ${ }^{3}$ Cambridge University Health, Judge Business School, Cambridge University, Cambridge, UK

Correspondence to: Laura M Heaton, US Census Bureau, 4600 Silver Hill Road, Washington, USA; Laura.M. Heaton@census.gov

Accepted 19 May 2008

\section{ABSTRACT}

Objective: To propose a methodology to estimate the number of new HIV infections averted. Knowledge of HIV infection has increased tremendously and modelling tools to project current epidemics into the future have greatly improved. Different types of models can be used to estimate HIV infections averted, although the number of new HIV infections averted cannot be measured directly. Method: Using cohort-component population projections, a disease modelling-based approach was used to compare the observed epidemiology of a disease after programme initiation with an expected epidemiology from past trends before programme initiation. The concept of modelling infections averted in a disease modelling-based approach involves a comparison between an "expected" or baseline epidemic with an "estimated" one. A hypothetical example was featured in order to demonstrate the proposed methodology. Using both the Estimation and Projection Package (EPP) and the Spectrum demographic modelling program, the underlying annual incidence levels implied by both the baseline and estimated epidemics were examined.

Results: The difference between baseline and estimated incidence levels is interpreted as "infections averted". Strengths and limitations of the approach are discussed. Conclusions: In this study an expected epidemiological approach was compared to one based on observation. Once sufficient data become available, the validation of various country data including HIV prevalence, mortality, and behaviour must be done. Additional information related to behaviour change may be critical to further support arguments for a change in disease trend. It is therefore important to use all available data, consequently strengthening findings from a disease modelling-based approach on HIV infections averted.

At present, the number of people living with HIV and AIDS continues to increase in many areas of the world. At the same time, we have the HIV prevention and treatment strategies and tools to mitigate the epidemic. To meet the challenges ahead we must continue to strengthen these. Effective prevention and treatment activities go towards averting potential HIV infections that would otherwise occur. Voluntary counselling and testing, condom social marketing, antiretroviral use and mass media campaigns are only a few of many strategies employed to curb HIV transmission.

In order to gauge the possible impacts of prevention and treatment programmes, a methodology must be in place to estimate HIV infections averted. In this paper, we introduce three basic approaches to estimate HIV infections averted. One of the three approaches, a disease modellingbased approach, is discussed at length.

\section{Coverage-based approach}

In this approach, estimation of new infections averted involves estimating the possible outcome of a proposed intervention. This estimate is typically based on a literature review and an assumption of the intervention's coverage. Evaluating the impact of a health intervention on disease is of great interest in the field of public health. One reason for this interest is to advocate for certain intervention programmes to reduce or halt the spread of a disease. Estimating the number of infections averted attributable to such programmes can also provide impetus to establishing the program, help focus programme activities, and set goals. ${ }^{1-9}$ Determining how much to scale up programmes to reach a larger number of people in need of the intervention is also of concern. ${ }^{10-12}$ An analysis of this approach is hypothetical in that we are answering questions such as "If male circumcision rates increase by $100 \%$ over a specific period of time, what are the possible number of HIV infections that might be averted over that time period?" Thus, the coverage-based approach can be valuable for public health advocacy purposes.

\section{Behaviour-based approach}

HIV infections are mediated by behaviour with interventions intended to change risky behaviours. A model can be constructed to evaluate these interventions for their effectiveness. This is known as a "behaviour-based approach", as HIV infections are mediated by behaviour with interventions aimed at changing risky behaviours. ${ }^{13-15}$ Most available interventions intend to reduce risky behaviours, thereby reducing new infections. Thus, if the effect of a behaviour change on new infections and the prevalence of changed behaviour are known (or assumed), mathematical models can be constructed based on behaviour. However, the relation between behaviour and disease is complex and dependent on the epidemiological context. ${ }^{15} 16$ Cost-effectiveness analysis of intervention programmes often occurs in both the coverage-based and behaviour-based approaches when selecting or advocating for a certain intervention. ${ }^{4-9} 111314$ A major problem with the behaviour-based approach is that there is a clear lack of relevant behavioural data to use in many developing countries, although there have been an increasing number of behavioural surveys. Therefore, the behaviour-based approach is difficult to implement currently in any reliable way in most developing countries.

\section{Disease modelling-based approach}

In this approach, the observed epidemiology of a disease after intervention can be compared with its past epidemiology or an expected epidemiology 
based on the past trend. This approach has been used to demonstrate the effect of a vaccination programme by showing a secular trend before and after introduction of the programme. ${ }^{17}$

In all three approaches, coverage, disease and behaviour, the number of infections averted needs to be estimated through mathematical modelling since it cannot be measured directly (that is, by definition, it is a non-event). Many studies in the coverage-based and behaviour-based approaches focus on the hypothetical analysis of the effect of certain interventions on disease trends. However, reliance on actual epidemiological change and an attempt to directly estimate infections averted based on the actual epidemiology makes the disease modellingbased approach preferable. A disease modelling-based approach for HIV infections averted is inherently theoretical, unlike control of endemic disease through biomedical solutions such as immunisation. It is of special importance, therefore, to validate and alter baseline curves based on the best available data in a disease modelling-based approach.

In this paper, we focus on the disease modelling-based approach to estimating new HIV infections averted. In brief, baseline projections of HIV incidence are prepared for countries using data pertaining to the time period before intervention programmes were instituted. This baseline will serve as a reference for future comparisons. In this paper, we use data for years through 2004 to derive the baseline projection. ${ }^{18}$ Subsequently, the incidence trend will be re-estimated for countries using additional epidemiological surveillance data available for years post-2004. The re-estimated incidence trend, compared to the baseline trend, will represent the change in the epidemic that may be the result of a number of factors including programme changes. The difference in the number of new HIV infections implied by the prevalence levels will be taken as the number of infections averted.

We examined several countries with sentinel surveillance data for years 2005 and 2006 and found that in the case of one of those countries, only a few thousand HIV infections were averted by 2010 . In another country, no new HIV infections were averted. Still, in another country, there were more infections in the re-estimated trend than there were in the baseline projection. To properly estimate infections averted, we need a sufficient number of years' worth of data post-2004 to gauge impacts. The major obstacle in carrying out this methodology with country-specific data is that insufficient sentinel surveillance data are available beyond 2004 to make this feasible at present. Validation of the results of the methodology proposed in this paper must await further data. Therefore, we demonstrate this model by illustrating a hypothetical example. Applications of such a modelling approach are discussed along with issues relevant to using this approach.

\section{METHODOLOGY}

The modelling process begins with undertaking an extensive review of data that contain information on epidemiology, population, fertility, mortality and migration. Sources include census and survey data, refugee and labour migration statistics, vital registration statistics, administrative records and HIV surveillance data. Once this review is complete, the new information is reconciled with the historical information. A decision is then made on whether a revision of previous population estimates and projections is warranted.

Although it has been clear for a number of years that mortality estimates and projections for many countries would have to be revised because of AIDS mortality, the lack of accurate empirical data on AIDS deaths, the paucity of data on HIV infection among the general population and the absence of tools to project the impact of AIDS epidemics into the future have all hampered these efforts. Although the accuracy of data on AIDS cases and AIDS deaths has not substantially improved, our knowledge of HIV infection has increased tremendously and modelling tools to project current epidemics into the future have greatly improved.

\section{Development of adult HIV prevalence estimates}

The Estimation and Projection Package (EPP) computer software is used to estimate and project adult (15-49) HIV prevalence from sentinel surveillance data in countries with generalised epidemics, defined as an epidemic with nationallevel prevalence above $1 \% .{ }^{19-21}$

In countries with a generalised epidemic, national estimates of HIV prevalence are typically based on data generated by surveillance systems that focus on pregnant women who attend a sample of sentinel antenatal clinics. Typically, an urban and a rural epidemic are derived, as the epidemics are normally distinct in most settings. The EPP software will then aggregate these two sub-epidemics to the national level. The national level results are used to obtain HIV prevalence and incidence curves used in estimation of HIV infections averted.

This method assumes that prevalence among pregnant women is a good approximation of prevalence among the adult population aged 15-49 in countries with a generalised HIV epidemic. $^{22}$ National population-based studies that include HIV testing have been completed in several countries in recent years. ${ }^{23-}$ ${ }^{25}$ Comparison of these results with those from the antenatal clinic data has provided evidence that the use of antenatal clinic data to model the epidemic among the adult population is appropriate. ${ }^{22} 23$ 26-28 There are some recent examples of considerably lower national HIV prevalence from population-based surveys, such as in Kenya. Results from these population-based surveys can be used in the EPP software to calibrate the urban and rural curves of HIV prevalence based on antenatal data to a nationally representative total prevalence level. ${ }^{20}$

\section{Development of the baseline projection}

Several stages are involved in the development of the baseline projection. First, hypothetical demographic estimates are prepared that do not include AIDS mortality. The impact of the HIV epidemic is then modelled. Estimates of AIDS mortality are combined with the estimates of non-AIDS mortality to obtain the baseline demographic estimates.

Hypothetical demographic results that do not include AIDSrelated mortality are produced using the Spectrum modelling program (The Futures Institute, Glastonbury, CT, USA).

The hypothetical series shows what would have happened if a country had not been affected by the HIV/AIDS epidemic. This modelling takes into account not only lower death rates but also associated changes to a country's age-sex structure and, indirectly, the combined effects of lower mortality and changing population composition on demographic indicators. This series assumes the same fertility rates (based on observed data) as the series that incorporates HIV/AIDS. The non-AIDS results are then input into the AIDS impact module (AIM) of Spectrum along with the trend in HIV prevalence from EPP and other epidemiological parameters. The estimates of incidence needed to estimate infections averted is one of many outputs from the Spectrum modelling program.

For ease of use, we use the 2006 revision of the World Population Prospects from the United Nations Population Division available in Spectrum for the demographic inputs. ${ }^{29}$ 
Once the AIM module is run, the user has the incidence and prevalence numbers needed for the baseline projection. Many of the inputs used in a typical modelling exercise are those that are based on exhaustive research by the UNAIDS modelling group. ${ }^{19} 20223031$ This group updates its assumptions when additional empirical research becomes available.

\section{DEVELOPMENT OF THE NUMBER OF INFECTIONS AVERTED Hypothetical example}

The concept of modelling infections averted in a disease modelling-based approach involves a comparison between an "expected" or baseline epidemic with an "estimated" one. We examine the underlying annual incidence levels implied by both the expected and estimated epidemics. The difference in the incidence levels is interpreted as infections averted. We use the EPP and Spectrum models to compare fits of the data before any major HIV programme initiatives, the baseline epidemic, and after these initiatives have started, the estimated epidemic. Major programme initiatives by various donors began in 2004 . As HIV prevalence data become available for the post-2004 period it is necessary to repeat the steps outlined above for the baseline estimates to incorporate the new data. This will result in new estimates of the number of new HIV cases for each year from 2005 to 2010. The difference between the baseline estimates of incidence and the post-2004 estimates is assumed to represent the number of infections averted during the period of interest.

We model two scenarios-a baseline projection and an assumed 25\% decline in HIV adult prevalence from the 2004 level by 2010 .

In the hypothetical example of modelling infections averted, both the number of baseline HIV infections and the resulting incidence numbers are shown for 1995 to 2010 (fig 1).

We are primarily interested in infections averted after the introduction of prevention programmes, 2005 in this example (fig 2).

An estimate of infections averted after 2004, if we assume a $25 \%$ decline in HIV prevalence by 2010 , would be represented in the shaded area (fig 3 ). The cumulative total of the shaded area represents the number of new infections that were averted during the period from 2005 to 2010 .

\section{DISCUSSION}

The number of infections averted cannot be measured directly, but a disease modelling-based approach is a direct estimation based on actual data from disease surveillance systems. In contrast, a coverage-based approach is indirect estimation based on intervention coverage (hypothetical or measured) and its presumed or estimated effect on disease. Similarly, a behaviour-based approach is indirect estimation based on a change in prevalence of certain behaviours and the estimated effect on a disease.

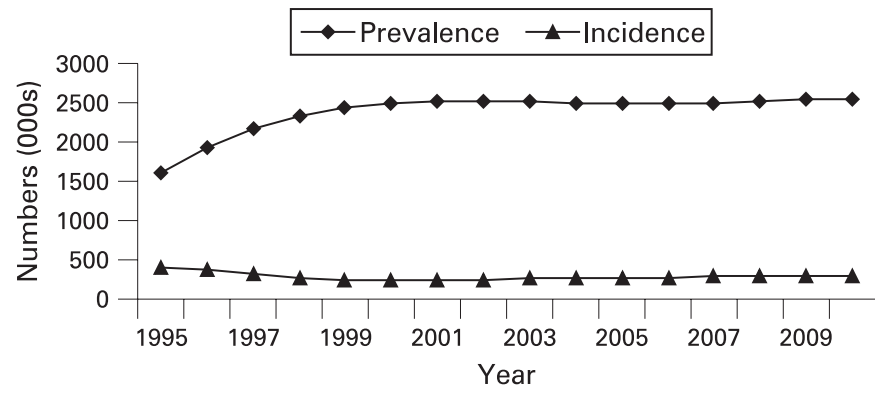

Figure 1 HIV prevalence and incidence baseline scenario: 1995-2010.

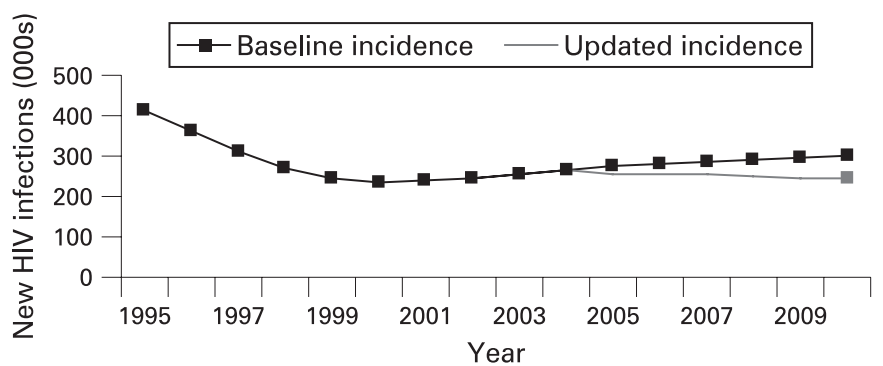

Figure 2 HIV incidence-two scenarios 1995-2010.

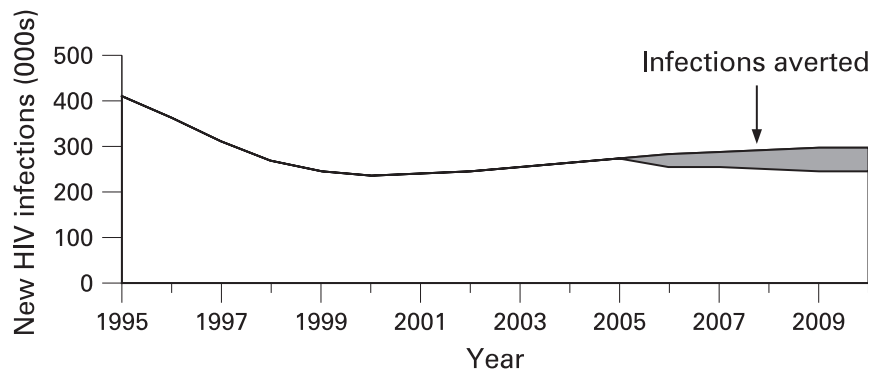

Figure 3 Cumulative number of HIV infections averted: 2005-10.

No approach can be exact since infections averted cannot be directly measured and any models are based on a number of assumptions. None the less, we propose a disease modellingbased approach in this paper to estimate infections averted because this approach is a more direct one. In addition, while we have described estimation of HIV infections averted for countries with generalised epidemics, this methodology can also be used to estimate infections averted for countries with concentrated epidemics as long as there are data to do so.

One use of estimating infections averted is to track progress made by international initiatives. The US President's Emergency Plan for AIDS Relief (PEPFAR) has a global goal of seven million HIV infections averted between the years 2005 and 2010, and a large amount of financial resources are disbursed in PEPFAR's 15 focus countries. Another global initiative, the Global Fund To Fight AIDS, Tuberculosis and Malaria, also has a large investment in HIV/AIDS programmes. In general, the impact of donor initiatives cannot be separated in a country. Indeed, prevention requires behavioural change along with enabling factors such as community activities, communication, commodity availability and a favourable legal and environmental framework. ${ }^{32-42}$ In the complex environment in which HIV prevention and treatment take place, it would be very difficult to assess a particular programme's impact.

Several issues related to the approach described in this paper need to be addressed. The first is related to the time lag in the processing and release of surveillance results. It will not be possible to make an estimate of infections averted until well after the post-2004 data are collected. We have observed this when applying the methodology to specific countries. In addition, the approach as described here can result in different historical levels of prevalence and incidence between the baseline scenario and the later scenario based on new HIV prevalence data that will be used to derive infections avertedthat is, when the new HIV prevalence data post-2004 are added to EPP, the software will often produce a somewhat different "best fit" of all the data, including the pre-2004 period. To address this issue, the EPP infections-averted fitting process can 
be adjusted so that the results covering the time period before the infections averted period approximate the original baseline results. This requires adjustment of any of the EPP fitting parameters-or some sort of ratio adjustment. Another way of addressing this issue is to limit new data entered into EPP to only the surveillance sites available at the time the baseline estimate was prepared (for example, only those sites available through 2003 or 2004). However, ignoring data from new sites added to a country's surveillance system at a later point in time may not be advisable. It will be important to validate the curves to HIV prevalence, mortality, and behavioural data from several sources once sufficient trend data are available.

Another concern is that of antiretroviral use. With widespread use of these drugs in resource-constrained settings, we could see an increase in prevalence, rather than a decline, that is not related to an increase in incidence. ${ }^{43}$ Although it appears that transmissibility of HIV may be reduced among people on antiretroviral treatment, this may be offset by increases in risktaking behaviour. For example, if people are feeling better as a result of antiretroviral treatment, they may engage in more sexual activity. However, studies have not been conclusive. Even in industrialised settings, there is no strong evidence that such disinhibition occurs. ${ }^{44}$ Therefore, the increase in prevalence due to increased survivorship of those on treatment needs to be factored out of the infections-averted estimation process. The next update of the EPP software will take antiretroviral use into account in order to better fit the models. ${ }^{45}$

Finally, in recent years, a number of countries have carried out population-based surveys that include HIV testing and many more are planned for the future. These surveys are finding different levels of HIV prevalence in the general population from that indicated by antenatal clinic sentinel surveillance. EPP allows for the national HIV prevalence curves to be calibrated to match the results of a population-based survey. At what point do results represent change due to programmes and not just a calibration to the time series? HIV prevalence itself has a range of uncertainty and this may also need to be addressed in infections averted. ${ }^{46-48}$

In a stable, endemic situation, a coverage-based approach may well produce convincing models as long as good knowledge of the relation between coverage and disease prevention exists. This is true for a measles vaccine, for instance. However, HIV epidemics are heterogeneous and the relation between coverage and disease prevention may not be the same in all cultural and epidemiological situations. ${ }^{49}$ Even with the behaviour-based approach, knowledge about behaviour change and infections averted is incomplete. Compared to stable endemic diseases, estimating the natural history of an increasing or decreasing epidemic is less straightforward, especially with limited surveillance data.

Infections averted are probably different for different levels of prevalence, but this often cannot be addressed or is only addressed in broad ways such as by differentiating by world regions in either a coverage-based or behaviour-based model. In stable, endemic situations, the disease modelling-based approach is simple and convincing, even without a good understanding of the intervention's impact on disease and the overall impact on the population, as long as the disease level clearly declines in the post-intervention period compared to an expected level. However, the HIV epidemic continues to evolve. While a coverage-based approach may be a better tool for advocacy and planning for an intervention programme, the disease modelling-based approach is better suited to track the possible effect of the intervention's actual implementation.

\section{Key messages}

- A disease modelling-based methodology has been proposed to estimate the number of HIV infections averted. It entails examining the number of new incident cases in an expected epidemic based on data from years before enactment of majo initiatives to the number of new incident cases in an actual epidemic using data after programme initiatives began.

- It is not possible to attribute the effects of the overall change in incident infections to specific programmes. Rather, an epidemic's trajectory can be analysed within the specific country context in relation to its overall programme activities and any behaviour change.

- Validation of results using empirical data from different countries needs to be done once sufficient data become available to do so.

Is integration of all three approaches a solution? Most probably it is. However, implementing integration is very difficult. The reality is far from the ideal owing to various gaps in data and limited scientific understanding and knowledge on the complicated and delicate relations between coverage, behaviour and disease. The Asian epidemic model (AEM) deals with both disease-related and behaviour-related information. ${ }^{50} 51$ It can produce better fitting models to the disease as well as the underlying behaviour and, consequently, scientifically convincing curves. Therefore, infections averted estimated by comparing two scenarios in the AEM application appear quite persuasive. However, data requirements are intensive and can be met successfully by only a few countries-for instance, Thailand. More extensive data collection should become routine as recommended by the World Health Organization and UNAIDS. $^{52}{ }^{53}$ Periodic analysis of collected data for strategic information purposes facilitates informed decision-making for programme management as well as evidence-based advocacy.

In conclusion, different types of models can be used to estimate HIV infections averted, but no model is perfect. We have delineated one approach here based on an expected epidemiology and an observed one, a conceptually simple approach, but some details still need careful consideration and further research. Moreover, it is important to improve the breadth and the availability of country level data on various aspects of the HIV epidemic and refine the models accordingly. The critical stage is to validate the curves to different country data sources including HIV prevalence, mortality and behaviour. Combining a coverage-based approach with a disease modelling-based approach is not simple because the two approaches have different sets of assumptions. Additional information related to behaviour change may be critical to have in some situations to further support arguments for a change in disease trend. ${ }^{54-64}$

Funding: Funding for the analysis and preparation of this manuscript was provided by The Global Fund To Fight AIDS, Tuberculosis and Malaria.

\section{Competing interests: None.}

Contributors: LH and RK were the lead authors of the manuscript. DLB, TF and PW provided input to the analysis and drafting of the manuscript. All authors reviewed the manuscript and approved its contents as well as providing significant input to the analysis.

\section{REFERENCES}

1. Baggaley RF, Garnett GP, Ferguson NM. Modelling the impact of antiretroviral use in resource-poor settings. PLoS Med 2006;3:493-504. 
2. Williams BG, Lloyd-Smith JO, Gouws E, et al. The potential impact of male circumcision on HIV in sub-Saharan Africa. PLoS Med 2006;3:1032-40.

3. Vickerman $\mathbf{P}$, Terris-Prestholt F, Delany $S$, et al. Are targeted HIV prevention activities cost-effective in high prevalence settings? Results from a sexually transmitted infection treatment project for sex workers in Johannesburg, South Africa. Sex Transm Dis 2006;33(suppl1):S122-32.

4. Teerawattananon $\mathbf{Y}$, Vos $T$, Tangcharoensathien V, et al. Cost-effectiveness of models for prevention of vertical HIV transmission—voluntary counseling and testing and choices of drug regimen. Cost Eff Resour Alloc 2005:3:7.

5. Soorapanth S, Sansom S, Bulterys M, et al. Cost-effectiveness of HIV rescreening during late pregnancy to prevent mother-to-child HIV transmission in South Africa and other resource-limited settings. J Acquir Immune Defic Syndr 2006;42:213-21.

6. Reynolds HW, Janowitz B, Homan R, et al. The value of contraception to prevent perinatal HIV transmission. Sex Transm Dis 2006;33:350-6.

7. Hogan DR, Baltussen $\mathrm{R}$, Hayashi $\mathrm{C}$, et al. Cost effectiveness analysis of strategies to combat HIV/AIDS in developing countries. BMJ 2005;331:1431-7.

8. Maclean CC, Stringer JSA. Potential cost-effectiveness of maternal and infant antiretroviral interventions to prevent mother-to-child transmission during breastfeeding. J Acquir Immune Defic Syndr 2005;38:570-7.

9. Sweat MD, O'Reilly KR, Schmid GP, et al. Cost-effectiveness of nevirapine to prevent mother-to-child HIV transmission in eight African countries. AIDS 2004;18:1661-71.

10. Stover J, Walker N, Garnett GP, et al. Can we reverse the HIV/AIDS pandemic with an expanded response? Lancet 2002;360:73-7.

11. Stover J, Bertozzi S, Gutierrez JP, et al. The global impact of scaling up HIV/AIDS prevention programs in low- and middle-income countries. Science 2006;311:14746 .

12. Salomon JA, Hogan DR, Stover J, et al. Integrating HIV prevention and treatment: from slogans to impact. PLoS Med 2005;2:50-6.

13. Vickerman $\mathbf{P}$, Kumaranayake $L$, Balakireva 0 , et al. The cost-effectiveness of expanding harm reduction activities for injecting drug users in Odessa, Ukraine. Sex Transm Dis 2006;33(suppl):S89-S102.

14. Kumaranayake L, Vickerman P, Walker D, et al. The cost-effectiveness of HIV preventive measures among injecting drug users in Svetlogorsk, Belarus. Addiction 2004;99:1565-76.

15. Grassly NC, Garnett GP, Schwartländer B, et al. The effectiveness of HIV prevention and the epidemiological context. Bull World Health Organ 2001;79:1121-35.

16. Fishbein M, Pequegnat W. Evaluating AIDS prevention interventions using behavioral and biological outcome measures. Sex Transm Dis 2000;27:101-10.

17. Ong G, Hoon HB, Ong A, et al. A 24-year review on the epidemiology and control of measles in Singapore, 1981-2004. Southeast Asian J Trop Med Public Health 2006;37:96-101.

18. US Census Bureau. Infections averted: concepts and an approach. Presented the UNAIDS/WHO Reference Group on Estimates, Modelling and Projections annual meeting, Athens, Greece, 2005.

19. The UNAIDS Reference Group on Estimates, Modelling and Projections Improved methods and assumptions for estimation of the HIV/AIDS epidemic and its impact: recommendations of the UNAIDS reference group on estimates, modelling and projections. AIDS 2002;16:W1-14.

20. Brown T, Grassly N, Garnett G, et al. Improving projections at the country level: the UNAIDS Estimation and Projection Package 2005. Sex Transm Infect 2006;82(Suppl 3):iii34-40.

21. Ghys PD, Brown T, Grassly NC, et al. The UNAIDS Estimation and Projection Package: a software package to estimate and project national HIV epidemics. Sex Transm Dis 2004:80(suppl1):i5-19.

22. Ghys PD, Kufa E, George MV. Measuring trends in prevalence and incidence of HIV infection in countries with generalised epidemics. Sex Transm Infect 2006;82(suppl I):i52-6.

23. García-Calleja JM, Gouws E, Ghys PD. National population based HIV prevalence surveys in sub-Saharan Africa: results and implications for HIV and AIDS estimates. Sex Transm Infect 2006;82(suppl 3):iii64-70.

24. García-Calleja JM, Marum LH, Cárcamo CP, et al. Lessons learned in the conduct, validation, and interpretation of national population based HIV surveys. AIDS 2005;19(suppl 2):S9-S17.

25. Mishra V, Vaessen M, Boerma JT, et al. HIV testing in national population-based surveys: experience from the demographic and health surveys. Bull World Health Organ 2006:84:537-45

26. Boerma JT, Ghys PD, Walker N. Estimates of HIV-1 prevalence from national population-based surveys as a new gold standard. Lancet 2003;362:1929-31.

27. Dzekedzeke K, Fylkesnes K. Reducing uncertainties in global HIV prevalence estimates: the case of Zambia. BMC Public Health 2006;6:83.

28. Fabiani M, Fylkesnes K, Nattabi B, et al. Evaluating two adjustment methods to extrapolate HIV prevalence from pregnant women to the general female population in sub-Saharan Africa. AIDS 2003;17:399-405.

29. United Nations. World population prospects, The 2006 revision. Department of Economic and Social Affairs. Population Division, 2007. http://www.un.org/esa/ population/publications/wpp2006/wpp2006.htm (accessed 30 Jan 2008).

30. Stover J, Walker N, Grassly NC, et al. Projecting the demographic impact of AIDS and the number of people in need of treatment: updates to the Spectrum projection package. Sex Transm Infect 2006;82(suppl 3):iii45-50.

31. Walker N, Stanecki KA, Brown T, et al. Methods and procedures for estimating HIV/ AIDS and its impact: the UNAIDS/WHO estimates for the end of 2001. AIDS 2003; 17:2215-25.
32. Bobrova N, Rhodes T, Power R, et al. Barriers to accessing drug treatment in Russia: a qualitative study among injecting drug users in two cities. Drug Alcohol Depend 2006;82 (suppl 1):S57-63

33. D'Cruz-Grote D. Prevention of HIV infection in developing countries. Lancet 1996;348:1071-4.

34. Hankins C. Changes in patterns of risk. AIDS Care 1998;10(suppl 2):S147-53.

35. Kerrigan D, Moreno L, Rosario S, et al. Environmental-structural interventions to reduce HIV/STI risk among female sex workers in the Dominican Republic. Am J Public Health 2006;96:120-5.

36. Latkin CA, Knowlton AR. Micro-social structural approaches to HIV prevention: a social ecological perspective. AIDS Care 2005;17(suppl 1):S102-13

37. Merson MH, Dayton JM, O'Reilly K. Effectiveness of HIV prevention interventions in developing countries. AIDS 2000;14(suppl 2):S68-84.

38. Morisky DE, Peña M, Tiglao TV, et al. The impact of the work environment on condom use among female bar workers in the Philippines. Health Educ Behav 2002;29:461-72.

39. Organista KC, Carrillo H, Ayala G. HIV prevention with Mexican migrants: review, critique, and recommendations. J Acquir Immune Defic Syndr 2004;37(suppl 4):S227-39.

40. Parker RG, Easton D, Klein CH. Structural barriers and facilitators in HIV prevention: a review of international research. AIDS 2000;14(suppl 1):S22-32.

41. Sweat MD, Denison JA. Reducing HIV incidence in developing countries with structural and environmental interventions. AIDS 1995;9(suppl A):S251-57.

42. Winch P. Social and cultural responses to emerging vector-borne diseases. J Vector Ecol 1998:23:47-53.

43. Boerma JT, Stanecki KA, Newell ML, et al. Monitoring the scale-up of antiretrovira therapy programmes: methods to estimate coverage. Bull World Health Organ 2006;84:145-50

44. Baggaley RF, Ferguson NM, Garnett GP. The epidemiological impact of antiretroviral use predicted by mathematical models: a review. Emerg Themes in Epidemiol 2005;2:9

45. Brown T, Salomon J, Alkema L, et al. Progress and challenges in modelling countrylevel HIV/AIDS epidemics: the UNAIDS Estimation and Projection Package 2007. Sex Transm Infect 2008;84(Suppl I):i5-i10.

46. Morgan M, Walker N, Gouws E, et al. Improved plausibility bounds about the 2005 HIV and AIDS estimates. Sex Transm Infect 2006;82 (suppl III):iii71-7.

47. Grassly NC, Morgan M, Walker N, et al. Uncertainty in estimates of HIV/AIDS: the estimation and application of plausibility bounds. Sex Transm Infect 2004;80/suppl I):i31-8.

48. Walker N, Grassly NC, Garnett GP, et al. Estimating the global burden of HIV/AIDS what do we really know about the HIV pandemic? Lancet 2004;363:2180-5.

49. Vickerman $\mathbf{P}$, Watts $\mathrm{C}$, Delany $\mathrm{S}$, et al. The importance of context: model projections on how microbicide impact could be affected by the underlying epidemiologic and behavioral situation in 2 African settings. Sex Transm Dis 2006;33:397-405

50. Mills S, Saidel T, Magnani R, et al. Surveillance and modelling of HIV, STI, and risk behaviours in concentrated HIV epidemics. Sex Transm Infect 2004;80(suppl 2):ii5762.

51. Brown T, Peerapatanapokin W. The Asian epidemic model: a process model for exploring HIV policy and programme alternatives in Asia. Sex Transm Infect 2004;80 (suppl I):i19-24.

52. UNAIDS/WHO Working Group on Global HIV/AIDS and STI Surveillance. Guidelines for second generation HIV surveillance. Geneva: WHO and UNAIDS, 2000.

53. Pisani E, Lazzari S, Walker N, et al. HIV surveillance: a global perspective. J Acquir Immune Defic Syndr 2003;32(suppl 1):S3-11.

54. Pervilhac C, Stover J, Pisani E, et al. Using HIV surveillance data: recent experiences and avenues for the future. AIDS 2005;19(suppl 2):S53-8.

55. Cleland J, Boerma JT, Carael M, et al. Monitoring sexual behaviour in general populations: a synthesis of lessons of the past decade. Sex Transm Infect 2004;80(suppl 2):ii1-7.

56. Zaba B, Slaymaker E, Urassa M, et al. The role of behavioral data in HIV surveillance AIDS 2005:19(suppl 2):S39-52.

57. Boerma JT, Weir SS. Integrating demographic and epidemiological approaches to research on HIV/AIDS: the proximate-determinants framework. J Infect Dis 2005; 191 (suppl 1):S61-7.

58. Hallett TB, Aberle-Grasse J, Bello G, et al. Declines in HIV prevalence can be associated with changing sexual behaviour in Uganda, urban Kenya, Zimbabwe, and urban Haiti. Sex Transm Infect 2006;82(suppl I):i1-8.

59. Hladik W, Shabbir I, Jelaludin A, et al. HIV/AIDS in Ethiopia: where is the epidemic heading? Sex Transm Infect 2006;82(suppl I):i32-5.

60. Bello GA, Chipeta J, Aberle-Grasse J. Assessment of trends in biological and behavioural surveillance data: is there any evidence of declining HIV prevalence or incidence in Malawi? Sex Transm Infect 2006;82(suppl I):i9-13.

61. Mahomva A, Greby S, Dube S, et al. HIV prevalence and trends from data in Zimbabwe, 1997-2004. Sex Transm Infect 2006;82(suppl I):i42-7.

62. Garnett GP, García-Calleja JM, Rehle T, et al. Behavioural data as an adjunct to HIV surveillance data. Sex Transm Infect 2006:82(suppl I):i57-62.

63. Stoneburner RL, Low-Beer D. Population level HIV declines and behavioral risk avoidance in Uganda. Science 2004;304:714-8 (erratum in Science 2004;306:1477)

64. Hallett TB, White PJ, Garnett GP. Appropriate evaluation of HIV prevention interventions: from experiment to full-scale implementation. Sex Transm Infect 2007;83(suppl I):i55-60. 